\title{
AVALIAÇÃO QUANTITATIVA DA SUSTENTABILIDADE ESTRUTURAL DE UM LATOSSOLO VERMELHO-AMARELO CULTIVADO COM EUCALIPTO NA REGIÃO DE PEÇANHA-MG ${ }^{1}$
}

\author{
Moacir de Souza Dias Junior², Fernando Palha Leite ${ }^{3}$, Mário Eugênio Winter ${ }^{4}$ e José Vicente Guimarães Pires ${ }^{5}$
}

\begin{abstract}
RESUMO - Uma das limitações para que o desenvolvimento florestal sustentável seja atingido está relacionado com a questão do tráfego durante a realização das operações de colheita e transporte de madeira, pois elas podem causar degradação da estrutura do solo. Visando obter uma solução para este problema, os objetivos deste estudo foram: a) propor um modelo de sustentabilidade da estrutura dos solos cultivados com Eucalyptus spp., na região de Peçanha-MG, em função da pressão de preconsolidação $\left(\sigma_{\mathrm{p}}\right)$ e da umidade $(\mathrm{U})$; e b) determinar, através do uso deste modelo, a influência das operações realizadas com o Feller-Büncher e Skidder (Pneu 30.5L.32), FellerBüncher e Skidder (Pneu 66.43.00.26) e Harvester e Forwarder na estrutura do solo. Este estudo foi conduzido em um Latossolo Vermelho-Amarelo (LV), sob Eucalyptus, utilizando amostras indeformadas, coletadas antes e após as operações da colheita, na profundidade de 0,10 a $0,125 \mathrm{~m}$. As amostras indeformadas foram utilizadas nos ensaios de compressão uniaxial. Foram determinadas também a textura e a densidade de partículas do solo. O modelo de sustentabilidade da estrutura do Latossolo Vermelho-Amarelo obtido para os projetos deste estudo é expresso pela seguinte equação: $\sigma_{\mathrm{p}}=10^{(2,72-1,29 \mathrm{U})}$. $\mathrm{O}$ uso dos modelos de sustentabilidade da estrutura e da pressão de preconsolidação, determinada após o tráfego, permitiu quantificar os efeitos causados pelo Feller-Büncher e Skidder (Pneu 30.5L.32), Feller-Büncher e Skidder (Pneu 66.43.00.26) e Harvester e Forwarder na estrutura do Latossolo Vermelho-Amarelo. Nos projetos Buriti e São Leonardo, as operações realizadas com o Harvester e Forwarder foram as que mais degradaram a estrutura do solo, enquanto no projeto Dourado foram as realizadas com o Feller-Büncher e Skidder (Pneu 30.5L.32) e Feller-Büncher e Skidder (Pneu 66.43.00.26).
\end{abstract}

Palavras-chave: Compactação, pressão de preconsolidação, Eucalyptus spp., sustentabilidade e colheita florestal mecanizada.

\section{QUANTITATIVE EVALUATION OF THE STRUCTURAL SUSTAINABILITY OF A RED-YELLOW LATOSOL CULTIVATED WITH EUCALYPTUS IN PEÇANHA-MG}

\begin{abstract}
One of the limitations for sustainable forest development is related to wheel traffic during harvest operations and wood transport, which can cause soil structure degradation. Seeking a solution for this problem, this study aimed to: a) propose a sustainability model of the structure of the soils cultivated with Eucalyptus spp., in Peçanha-MG as a function of the preconsolidation pressure $\left(\sigma_{p}\right)$ and moisture content $(U)$ and b) determine, throught the use of this model, the effect of the harvest operations using Feller Büncher and Skidder (tire 30.5L.32), Feller Buncher and Skidder (tire 66.43.00.26) and Harvester and Forwarder on the soil structure. This study was conducted on a Red-Yellow Latosol under Eucalyptus, using undisturbed soil samples collected before and after harvest operations at 0.10-0.125 $\mathrm{m}$ depth. The undisturbed soil samples were used in the uniaxial compression tests. Particle size distribution and particle density were also determined. The soil sustainability model for the

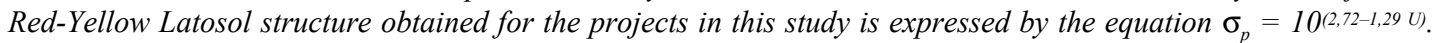
The use of the soil structure sustainability models and the preconsolidation pressure determined after wheel
\end{abstract}

1 Recebido para publicação em 18.4.2000.

Aceito para publicação em 12.5.2003.

2 Professor do Departamento de Ciência do Solo da Universidade Federal de Lavras - UFLA, Bolsista do CNPq, <msouzadj@ufla.br>. ${ }^{3}$ Eng.-Agr., Dr - CENIBRA, <fernando@cenibra.com.br>; ${ }^{4}$ Engenheiro Mecânico - CENIBRA;

${ }^{5}$ Engenheiro Florestal - CENIBRA. 
traffic allowed to quantify the effects of Feller-Büncher and Skidder (tire 30.5L.32), Feller-Büncher and Skidder (tire 66.43.00.26) and Harvester and Forwarder on the Red-Yellow Latosol structure. In the Buriti and São Leonardo Projects, the operations with Harvester and Forwarder degraded the soil structure the most, while in the Dourado Project the operations with Feller-Büncher and Skidder (tire 30.5L.32) and Feller-Büncher and Skidder (tire 66.43.00.26) caused more soil structure degradation.

Key words: $\quad$ Soil compaction, preconsolidation pressure, Eucalyptus spp., sustainability and mechanical operations.

\section{INTRODUÇÃO}

Uma das limitações para que o desenvolvimento florestal sustentável seja atingido está relacionada com a questão do tráfego de máquinas e veículos durante a realização das operações de colheita e transporte da madeira (Dias Junior et al., 1999). Este tráfego pode causar degradação da estrutura do solo em função, às vezes, do excesso de umidade ou capacidade de suporte de carga do solo restrita no momento de realização das operações. $\mathrm{O}$ tráfego indiscriminado nas áreas florestadas tem sido preocupante por causa da possibilidade de disseminação da compactação, pois esta pode causar redução na produtividade. Assim, torna-se importante determinar a umidade e a capacidade de suporte de carga dos solos, para que danos à sua estrutura possam ser evitados (Dias Junior, 1994; Dias Junior \& Pierce, 1996).

A preocupação com a compactação do solo se justifica pelo fato de os pesquisadores terem demonstrado que ela aumenta a densidade e a resistência mecânica do solo (Hillel, 1982; Wagger \& Denton, 1989; Hill \& MezaMontalvo; 1990; Lebert \& Horn, 1991) e diminui a porosidade total e o tamanho e a continuidade dos poros (Hillel, 1982; Smucker \& Erickson, 1989). Reduções significativas ocorrem principalmente no volume dos macroporos, enquanto os microporos permanecem praticamente inalterados (Hillel, 1982). A compactação do solo pode limitar a adsorção e, ou, absorção de nutrientes, a infiltração e a redistribuição de água, as trocas gasosas e o desenvolvimento do sistema radicular (Grohmann \& Queiroz Neto, 1966; Alvarenga et al., 1983; Smucker \& Erickson, 1989; Bicki \& Siemens, 1991), resultando em decréscimo da produção e aumento da erosão e da potência necessária para o preparo do solo (Soane, 1990).

Conhecidos os problemas que podem ser causados ao solo devido ao seu manejo inadequado, uma alternativa para enfrentá-los é a prevenção do processo de compactação. Assim, para se ter uma silvicultura sustentável, é

R. Árvore, Viçosa-MG, v.27, n.3, p.343-349, 2003 importante conhecer a capacidade de suporte de carga do solo no momento das operações silviculturais, para que esta possa nortear o planejamento destas operações, a fim de evitar a compactação do solo.

Visando obter uma solução para o problema, os objetivos deste estudo foram: a) propor um modelo de sustentabilidade da estrutura de um Latossolo VermelhoAmarelo cultivado com Eucalyptus spp., na região de Peçanha - MG, em função da pressão de preconsolidação $\left(\sigma_{\mathrm{p}}\right)$ e da umidade (U); e b) determinar, através do uso deste modelo, a influência das operações realizadas com o Feller-Büncher e Skidder (Pneu 30.5L.32), FellerBüncher e Skidder (Pneu 66.43.00.26) e Harvester e Forwarder, na estrutura do solo na profundidade de 0,10 a $0,125 \mathrm{~m}$.

\section{MATERIAL E MÉTODOS}

Este estudo foi conduzido em áreas experimentais situadas no município Peçanha - MG. Utilizou-se um Latossolo Vermelho-Amarelo (LV), sob cultivo de Eucalyptus spp., do qual foram obtidas amostras indeformadas da profundidade de 0,10 a $0,125 \mathrm{~m}$, por ter sido esta a profundidade que apresentou, em estudo preliminar, a maior resistência à penetração. A estrutura da camada superficial é granular e sua mineralogia é basicamente constituída de quartzo nas frações areia e silte e caulinita, gibbsita, goethita e hematita (em menor proporção) na fração argila. A amostragem foi realizada nos projetos Buriti, Dourado e São Leonardo. Estes projetos encontravam-se ao final do primeiro ciclo de cultivo com Eucalyptus, e as amostragens foram obtidas em povoamentos nas idades de 9,6, 10,6 e 12,6 anos nos projetos Buriti, Dourado e São Leonardo, respectivamente. As máquinas utilizadas na colheita florestal foram Feller-Büncher de disco, modelo 2618 com rodante de esteiras; Skidder (Pneu 30.5L.32) tração 4x4, modelo 460; Skidder (Pneu 66.43.00.26) tração 4x4, modelo 460; 
Harvester de pneus, tração 6x6, modelo 1270; e Forwarder de pneus, tração 8x8, modelo 1710 .

O sistema de amostragem consistiu de duas etapas.

\subsection{Antes do Tráfego das Operações de Colheita}

Nos projetos Buriti, Dourado e São Leonardo, foram coletadas aleatoriamente, em cada projeto, 36 amostras indeformadas (diâmetro $6,4 \mathrm{~cm}$ e $2,54 \mathrm{~cm}$ de altura), na profundidade de 0,10 a $0,125 \mathrm{~m}$, utilizando o amostrados de Uhland. Para obter a relação entre $\sigma_{\mathrm{p}} \mathrm{e} U$, as amostras foram inicialmente saturadas e secas ao ar no laboratório e, em seguida, submetidas ao ensaio de compressão uniaxial de acordo com Bowles (1986). As saturações das amostras foram feitas ao adicionar água até dois terços de sua altura durante 24 horas e, em seguida, estas foram secas em laboratório, até obter a faixa de umidade de 0,02 a $0,67 \mathrm{~kg} / \mathrm{kg}$. Esta faixa de umidade foi obtida através do controle do peso úmido das amostras. Após a obtenção destas umidades, as amostras foram submetidas ao ensaio de compressão uniaxial, usando um consolidômetro da marca Boart Longyear, cuja aplicação das pressões foi feita através de ar comprimido. As pressões aplicadas a cada amostra obedeceram à seguinte ordem: 25, 50, 100, $200,400,800$ e $1.600 \mathrm{kPa}$. Cada pressão foi aplicada até que $90 \%$ da deformação máxima fosse atingida (Taylor, 1948). Após esta condição ser alcançada, aplicou-se nova pressão. Depois da realização do ensaio, as $\sigma_{\mathrm{p}}$ foram obtidas de acordo com Dias Junior \& Pierce (1995), usando as curvas de compressão do solo. Em seguida, as $\sigma_{\mathrm{p}}$ foram plotadas em função da U. Através do uso do software Sigma Plot 4.0, foram ajustadas as equações que correspondem aos modelos de sustentabilidade da estrutura com os respectivos intervalos de confiança da população, a 95\%. Os intervalos de confiança da população foram utilizados para avaliar e confrontar com as $\sigma_{\mathrm{p}}$ obtidas de outras populações diferentes daquela usada na obtenção do modelo de sustentabilidade da estrutura. Os modelos de sustentabilidade da estrutura foram ajustados para cada projeto, de acordo com a equação $\sigma_{p}=10^{(a+b u)}$, sendo "a" e "b" parâmetros estimados. Foram determinadas também a textura (Day, 1986) e a densidade de partículas (Blake \& Hartge, 1986).

\subsection{Após as Operações Mecanizadas da Colheita}

Nos projetos Buriti, Dourado e São Leonardo, 36 amostras indeformadas (diâmetro $6,4 \mathrm{~cm}$ e 2,54 cm de altura) foram coletadas em cada projeto, na profundidade de 0,10 a $0,125 \mathrm{~m}$, utilizando o amostrador de Uhland. Estas amostras foram coletadas na linha de tráfego do Feller-Büncher e Skidder (Pneu 30.5L.32); Feller-Büncher e Skidder (Pneu 66.43.00.26) e Harvester e Forwarder, com a finalidade de quantificar os efeitos destas operações sobre a estrutura do solo através da $\sigma_{\mathrm{p}}$. Essas amostras foram submetidas ao ensaio de compressão uniaxial, como descrito anteriormente, com a umidade na qual as operações mecanizadas foram realizadas. Através das curvas de compressão, as $\sigma_{\mathrm{p}}$ induzidas pelo tráfego foram determinadas.

Para não ocorrer alteração na umidade desde o momento da coleta até o da realização do ensaio de compressão uniaxial no laboratório, as amostras indeformadas foram parafinadas no campo e enviadas, em seguida, para o laboratório de Física do Solo da Universidade Federal de Lavras.

Neste estudo usou-se $\sigma_{\mathrm{p}}$ como uma medida da capacidade de suporte de carga dos solos parcialmente saturados (Dias Junior, 1994) e como um indicador da sustentabilidade da estrutura (Dias Junior et al., 1999).

As análises de regressões foram realizadas com o software Sigma Plot 4.0 (Jandel Scientific), e a comparação das regressões foi feita segundo o procedimento descrito em Snedecor \& Cochran (1989). Este procedimento permite testar a homogeneidade dos dados através do teste $\mathrm{F}$. Se este teste for não-significativo, as equações são homogêneas, caso contrário, as equações são diferentes.

\section{RESULTADOS E DISCUSSÃO}

O LV do projeto São Leonardo apresentou teor de areia maior que os dos projetos Buriti e Dourado (Quadro 1). Considerando que a densidade máxima do solo aumenta com o incremento do teor de areia do solo (Dias Junior \& Estanislau, 1999), observa-se que o LV do projeto São Leonardo apresentou maior densidade do solo antes do tráfego do que o projeto Dourado (Quadro 1). Os valores da densidade do solo antes do tráfego seguiram a seguinte ordem: densidade do solo do projeto São Leonardo $=$ densidade do solo do projeto Buriti > densidade do solo do projeto Dourado.

As operações realizadas com o Feller-Büncher e Skidder (Pneu 30.5L.32), Feller-Büncher e Skidder (Pneu 66.43.00.26) aumentaram a densidade do solo inicial

R. Árvore, Viçosa-MG, v.27, n.3, p.343-349, 2003 
somente no projeto Dourado, enquanto as operações realizadas com o Harvester e Forwarder aumentaram as densidades do solo inicial nos projetos Buriti e Dourado. Portanto, as operações realizadas com o Harvester e Forwarder afetaram as densidades iniciais em dois terços dos projetos estudados (Quadro 2).

Os modelos de sustentabilidade da estrutura do LV para a profundidade de 0,10 a $0,125 \mathrm{~m}$, para os projetos Buriti, Dourado e São Leonardo, estão apresentados na Figura 1. Estas equações foram as mesmas encontradas por Dias Junior, (1994), as quais apresentaram $\mathrm{R}^{2}$ significativos a $1 \%$ e iguais a 0,95 e 0,97 .

As equações de regressões entre $\sigma_{\mathrm{p}}$ e $\mathrm{U}$ dos projeto Buriti, Dourado e São Leonardo foram homogêneas, indicando a mesma capacidade de suporte de carga na profundidade de 0,10 a $0,125 \mathrm{~m}$ dos três projetos. Portanto, uma nova equação de regressão foi ajustada a todos os valores de $\sigma_{\mathrm{p}}$ e $\mathrm{U}$, obtendo-se um único modelo de sustentabilidade da estrutura para o Latossolo VermelhoAmarelo. O modelo obtido é expresso pela seguinte

Quadro 1 - Caracterização física do solo (profundidade de 0,10 a $0,125 \mathrm{~m}$ )

Table 1 - Physical characterization of the soil (0.10 to $0.125 \mathrm{~m} \mathrm{depth)}$

\begin{tabular}{|l|c|c|c|c|c|}
\hline \multirow{2}{*}{ Projeto } & Argila & Silte & Areia & Dsi $^{1 /}$ & $\mathrm{Dp}^{2 /}$ \\
\cline { 2 - 6 } & \multicolumn{4}{|c|}{$(\mathrm{g} / \mathrm{kg})$} & \multicolumn{2}{|c|}{$\left(\mathrm{kg} / \mathrm{dm}^{3}\right)$} \\
\hline Buriti & $500^{\frac{3 /}{} \mathrm{a}}$ & $80^{\frac{3}{2}} \mathrm{a}$ & $420^{\frac{3}{-}} \mathrm{b}$ & $1,02^{4 /} \mathrm{a}$ & $2,63^{\frac{3}{}}$ \\
Dourado & $500 \mathrm{a}$ & $90 \mathrm{a}$ & $410 \mathrm{~b}$ & $0,89 \mathrm{~b}$ & 2,53 \\
São Leonardo & $400 \mathrm{a}$ & $90 \mathrm{a}$ & $510 \mathrm{a}$ & $1,04 \mathrm{a}$ & 2,46 \\
\hline
\end{tabular}

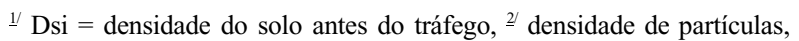

${ }^{3 /}$ média de três repetições e ${ }^{4}$ média de 36 repetições.

Médias seguidas de mesma letra, nas colunas, não diferem entre si pelo teste de Scott-Knott, a 5\%. equação: $\sigma_{\mathrm{p}}=10^{(2,72-1,29 \mathrm{U})}$, com $\mathrm{R}^{2}$ significativo a $1 \% \mathrm{e}$ igual a 0,93 . Este modelo fornece, portanto, a capacidade de suporte de carga do solo em termos da pressão de preconsolidação, que é uma estimativa da pressão máxima que pode ser aplicada ao solo para evitar que a compactação adicional ocorra.

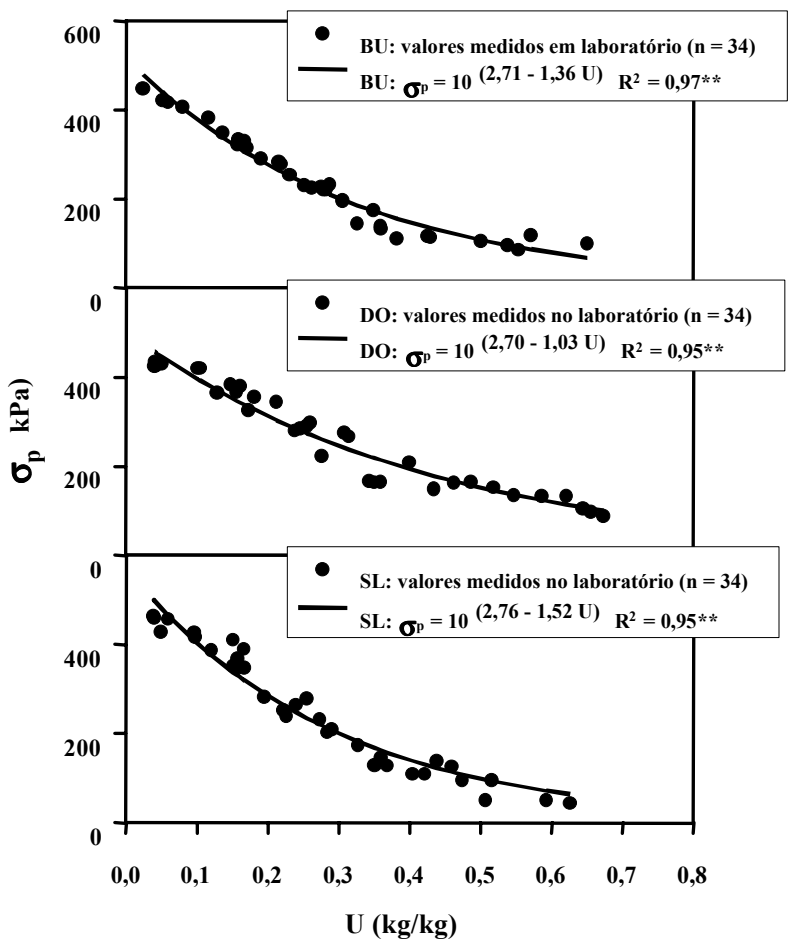

Figura 1 - Modelos de sustentabilidade da estrutura para o Latossolo Vermelho-Amarelo na profundidade de 0,10 a $0,125 \mathrm{~m}$ dos projetos Buriti (BU), Dourado (DO) e São Leonardo (SL).

Figure 1 - Sustainability models of the Red-Yellow Latosol structure for 0.10-0.125 m depth of Buriti (BU), Dourado (DO) and São Leonardo (SL) Projects.

Quadro 2 - Densidade do solo antes (Dsi) e após (Dst) as operações com Feller-Büncher e Skidder (Pneu 30.5L.32), FellerBüncher e Skidder (Pneu 66.43.00.26) e Harvester e Forwarder

Table 2 - Bulk density before (Dsi) and after (Dst) Feller-Büncher and Skidder (Tire 30.5L.32); Feller-Büncher and Skidder (tire 66.43.00.26) and Harvester and Forwarder operation

\begin{tabular}{|c|c|c|c|c|c|c|}
\hline \multirow{3}{*}{ Veículo } & \multicolumn{2}{|c|}{ Buriti } & \multicolumn{2}{|c|}{ Dourado } & \multicolumn{2}{|c|}{ São Leonardo } \\
\hline & $\mathrm{Dsi}^{1 /}$ & $\mathrm{Dst}^{2 /}$ & Dsi & Dst & Dsi & Dst \\
\hline & \multicolumn{6}{|c|}{$\left(\mathrm{mg} / \mathrm{m}^{3}\right)$} \\
\hline Feller-Büncher e Skidder (Pneu 30.5L.32) & $1,02^{\frac{3}{3}} \mathrm{a}$ & $1,03^{4 /} \mathrm{a}$ & $0,92^{3 /} \mathrm{b}$ & $0,97^{4 /} \mathrm{a}$ & $1,04^{3 /} \mathrm{a}$ & $1,12^{4 /} \mathrm{a}$ \\
\hline Feller-Büncher e Skidder (Pneu 66.43.00.26) & $1,02 \mathrm{a}$ & $1,00 \mathrm{a}$ & $0,92 \mathrm{~b}$ & 0,97 a & $1,04 \mathrm{a}$ & $1,08 \quad a$ \\
\hline Harvester e Forwarder & $1,02 \mathrm{~b}$ & 1,09 a & $0,92 \mathrm{~b}$ & $0,99 \mathrm{a}$ & 1,04 a & $1,12 \mathrm{a}$ \\
\hline
\end{tabular}

${ }^{1 /}$ Densidade do solo inicial, ${ }^{2 /}$ densidade do solo após o tráfego, ${ }^{3 /}$ média de 36 repetições e ${ }^{4 /}$ média de 12 repetições.

Médias seguidas de mesma letra, nas linhas e para cada projeto, não diferem entre si pelo teste de Scott-Knott, a $5 \%$. 
Seguindo a sugestão de Dias Junior et al. (1999), nos modelos de sustentabilidade da estrutura (Figura 1) foram plotados os valores das $\sigma_{\mathrm{p}}$ obtidas nas amostras indeformadas, que foram coletadas após as operações com o Feller-Büncher e Skidder (Pneu 30.5L.32), FellerBüncher e Skidder (Pneu 66.43.00.26) e Harvester e Forwarder (Figuras 2, 3 e 4). Nestas figuras, além dos modelos de sustentabilidade da estrutura obtidos antes das operações da colheita, apresenta-se também o intervalo de confiança da população a $95 \%$.

O tráfego efetuado com Feller-Büncher e Skidder (Pneu 30.5L.32), Feller-Büncher e Skidder (Pneu 66.43.00.26) e Harvester e Forwarder, em todos os projetos, resultou, em algumas amostras, na degradação da estrutura do solo (pontos acima da curva $\sigma_{\mathrm{p}}$ versus $U$ ), permitindo a identificação dos locais onde ocorreu a compactação do solo causada por estas operações

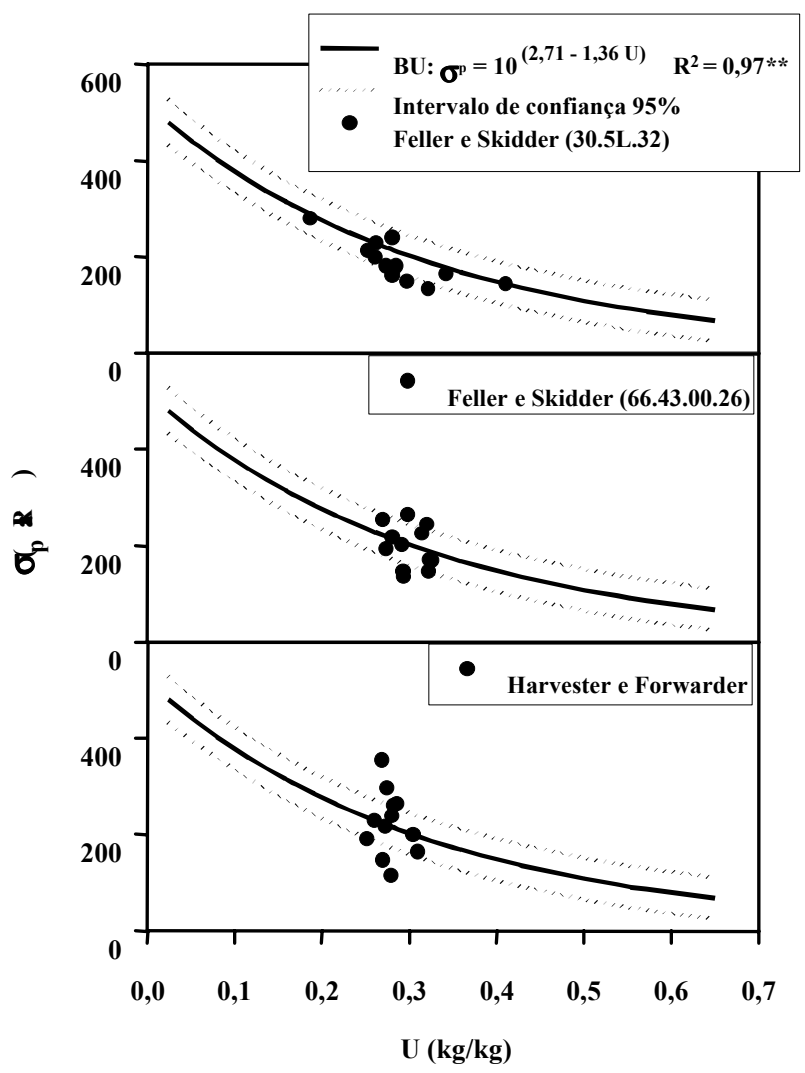

Figura 2 - Modelo de sustentabilidade da estrutura do Latossolo Vermelho-Amarelo na profundidade de 0,10 a $0,125 \mathrm{~m}$ do projeto Buriti (BU).

Figure 2 - Sustainability model of the Red-Yellow Latosol structure at 0.10-0.125 m depth of the Buriti Project (BU).
(Figuras 2, 3, e 4). A identificação das áreas compactadas torna-se importante, pelo fato de a compactação poder afetar a resistência mecânica do solo, o volume dos macroporos, a infiltração e redistribuição de água, as trocas gasosas e o desenvolvimento do sistema radicular, resultando em decréscimo da produção e aumento da erosão (Hillel, 1982; Wagger \& Denton, 1989; Hill E MezaMontalvo; 1990; Soane, 1990; Lebert \& Horn, 1991) e do custo final das operações de preparo do solo.

Nas Figuras 2, 3 e 4 e no Quadro 3, observa-se que nos projetos Buriti e São Leonardo as operações realizadas com o Harvester e Forwarder foram as que mais causaram compactação, enquanto no projeto Dourado as operações realizadas com o Feller-Büncher e Skidder (Pneu 30.5L.32) e com o Feller-Büncher e Skidder (Pneu 66.43.00.26) foram as que mais degradaram a estrutura do solo.

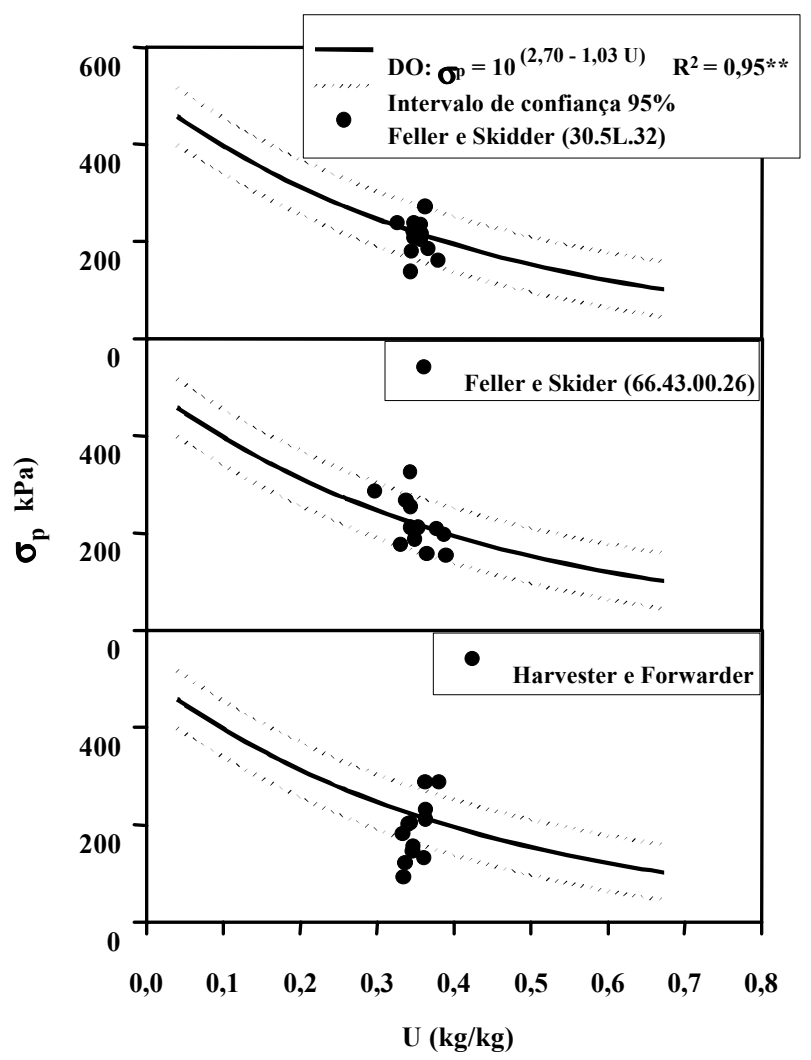

Figura 3 - Modelo de sustentabilidade da estrutura do Latossolo Vermelho-Amarelo na profundidade de 0,10 a $0,125 \mathrm{~m}$ do projeto Dourado.

Figure 3 -Structural sustainability model of the Red-Yellow Latosol at 0.10-0.125 $\mathrm{m}$ depth of the Dourado Project.

R. Árvore, Viçosa-MG, v.27, n.3, p.343-349, 2003 


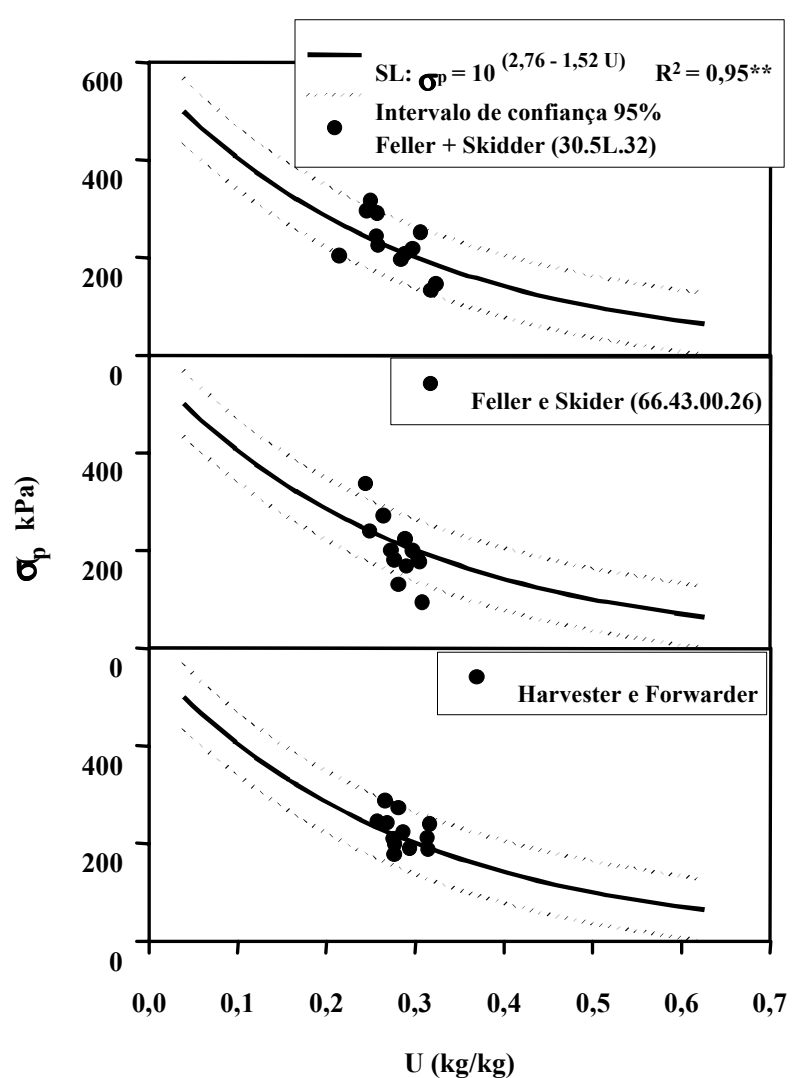

Figura 4 - Modelo de sustentabilidade da estrutura do Latossolo Vermelho-Amarelo na profundidade de 0,10 a $0,125 \mathrm{~m}$ do projeto São Leonardo (SL).

Figure 4 - Structural sustainability model of the Red-Yellow Latosol at 0.10-0.125 depth of the São Leonardo Project (SL).

Quadro 3 - Porcentagem de amostras com pressões de preconsolidação determinadas após as operações da colheita com o Feller-Büncher e Skidder (Pneu 30.5L.32), FellerBüncher e Skidder (Pneu 66.43.00.26) e Harvester e Forwarder

Table 3 - Percentage of soil samples with preconsolidation pressures determined after the harvest operations with Feller-Büncher and Skidder (Tire 30.5L.32), FellerBüncher and Skidder (Tire 66.43.00.26) and Harvester e Forwarder

\begin{tabular}{|l|c|c|c|c|}
\hline \multirow{2}{*}{ Veículo } & Buriti & Dourado & $\begin{array}{c}\text { São } \\
\text { Leonardo }\end{array}$ & Média \\
\cline { 2 - 5 } & \multicolumn{5}{|c|}{$\sigma^{\frac{1}{}>} \sigma_{\mathrm{pe}}^{\frac{2}{2}}$} \\
\hline $\begin{array}{l}\text { Feller-Büncher e Skidder } \\
\text { (Pneu 30.5L.32) }\end{array}$ & 8 & 33 & 50 & $30 \pm 21$ \\
$\begin{array}{l}\text { Feller-Büncher e Skidder } \\
\text { (Pneu 66.43.00.26) }\end{array}$ & 33 & 33 & 25 & $30 \pm 5$ \\
Harvester e Forwarder & 42 & 25 & 58 & $42 \pm 17$ \\
\hline
\end{tabular}

1' Pressão de preconsolidação determinada após as operações de colheita e ${ }^{2 /}$ pressão de preconsolidação estimada pelo modelo de sustentabilidade da estrutura.
Em média, as operações realizadas com o FellerBüncher e Skidder (Pneu 30.5L.32) e o Feller-Büncher e Skidder (Pneu 66.43.00.26) foram as que menos degradaram a estrutura do solo, enquanto as operações realizadas com o Harvester e Forwarder foram as que mais degradaram a estrutura do solo (Quadro 3). Apesar de as operações realizadas com o Harvester e Forwarder terem causado maior degradação da estrutura do solo, deve-se ressaltar que estes impactos são sistemáticos, abrangendo posições definidas e menor área, enquanto os causados pelas operações com o Feller-Büncher e Skidder não são sistemáticos, têm distribuição aleatória e abrangem maior área.

\section{CONCLUSÕES}

a) O modelo de sustentabilidade da estrutura do Latossolo Vermelho-Amarelo para os projetos deste estudo é expresso pela seguinte equação: $\sigma_{\mathrm{p}}=10^{(2,72-1,29 \mathrm{U})}$.

b) O uso dos modelos de sustentabilidade da estrutura e da pressão de preconsolidação, determinada após as operações de colheita, permitiu quantificar os efeitos causados pelo Feller-Büncher e Skidder (Pneu 30.5L.32), Feller-Büncher e Skidder (Pneu 66.43.00.26) e Harvester e Forwarder na estrutura do Latossolo VermelhoAmarelo.

c) Nos projetos Buriti e São Leonardo, as operações realizadas com o Harvester e Forwarder foram as que mais degradaram a estrutura do solo, enquanto no projeto Dourado foram as realizadas com o Feller-Büncher e Skidder (Pneu 30.5L.32) e Feller-Büncher e Skidder (Pneu 66.43.00.26).

\section{REFERÊNCIAS BIBLIOGRAFICAS}

ALVARENGA, R. C. et al. Armazenamento de água em um podzólico Vermelho-Amarelo sob pastagem e cultivo anual. Ciência e Prática, v. 7, p. 48-57, 1983.

BICKI, T. J.; SIEMENS, J. C. Crop response to wheel traffic soil compaction. Transaction of the American Society of Agricultural Engineers, v. 34, p. 909-913, 1991.

BLAKE, G. R.; HARTGE, K. H. Particle density. In: KLUTE, C. A. (Ed.) Methods of soil analysis. Part 1. Physical and mineralogical methods. 2.ed. Madison: American Society Agronomy, 1986. p. 377-382. (Agronomy Monogronomy, 9).

BOWLES, J. E. Engineering properties of soils and their measurements. Third edition. Auckland: McGraw-Hill, 1986. $218 \mathrm{p}$. 
DAY, P. R. Particle fraction and particle size analysis. In: KLUTE, C. A. (Ed.) Methods of soil analysis. Part 1. Physical and mineralogical methods. 2.ed. Madison: American Society Agronomy, 1986. p. 545-567.

(Agronomy Monogronomy, 9)

DIAS JUNIOR, M. S. Compression of three soils under long-term tillage and wheel traffic. 1994. $114 \mathrm{f}$. Tese (Doutorado Crop and Soil Science) - Michigan State University, East Lansing, 1994.

DIAS JUNIOR, M. S. et al. Avaliação quantitativa da sustentabilidade da estruturados solos em sistemas florestais na região de Aracruz-ES. Revista Árvore, v. 23, p. 371$380,1999$.

DIAS JUNIOR, M. S.; ESTANISLAU, W. T. Grau de compactação e retenção de água de Latossolos submetidos a diferentes sistemas de manejo. Revista Brasileira de Ciência do Solo, v. 23, p. 45-51, 1999.

DIAS JUNIOR, M. S.; PIERCE, F. J. A simple procedure for estimating preconsolidation pressure from soil compression curves. Soil Technology, v. 8, p. 139-151, 1995.

DIAS JUNIOR, M. S.; PIERCE, F. J. Revisão de Literatura. O processo de compactação do solo e sua modelagem.

Revista Brasileira de Ciência do Solo, v. 20, p. 175-182, 1996.

GROHMANN, F.; QUEIROZ NETO, J. P. Efeito da compactação artificial de dois solos limo-argilosos sobre a penetração das raízes de arroz. Bragantia, v. 25, p. 421431, 1966.
HILL, R. L.; MEZA-MONTALVO, M. Long-term wheel traffic effects on soil physical properties under different tillage systems. Soil Science Society of America Journal, v. 54, p. 865-870, 1990.

HILLEL, D. Introduction to soil physics. San Diego: Academic Press, 1982. 364 p.

LEBERT, M.; HORN, R. A method to predict the mechanical strength of agricultural soils. Soil \& Tillage Research, v. 19, p. 274-286, 1991.

SMUCKER, A. J. M.; ERICKSON, A. E. Tillage and compactive modifications of gaseous flow and soil aeration. In: LARSON, W. E.; BLAKE, G. R.; ALLMARAS, R. R.; VOORHEES, W. B.; GUPTA, S. C. (Eds.). Mechanics related process in structured agricultural soils. The Netherlands, NATO Applied Science, 1989. v. 172, p. 205221.

SNEDECOR, G. W.; COCHARAN. Statistical methods. \&. ed. Ames: Iowa State University Press, 1989. 503 p.

SOANE, B. D. The role of organic matter in soil compactibility: a review of some practical aspects. Soil $\boldsymbol{\&}$ Tillage Research, v. 16, p. 179-201, 1990.

TAYLOR, D. W. Fundamentals of soil mechanics. New York: John Wiley \& Sons, 1948. 770 p.

WAGGER, M.G.; DENTON, H. P. Influence of cover crop and wheel traffic on soil physical properties in continuous no-till corn. Soil Science Society of American Journal, v. 53, p. 1206-1210, 1989. 